\title{
End-of-life care in an Australian acute hospital: a retrospective observational study
}

\author{
Ninya Maubach, ${ }^{1}$ Monique Batten, ${ }^{1}$ Scott Jones, ${ }^{1}$ Judy Chen, ${ }^{1}$ Brett Scholz $\mathbb{1 0},{ }^{1}$ Alison Davis, ${ }^{1,2}$ \\ Jonathan Bromley, ${ }^{1,2}$ Brandon Burke, ${ }^{1,2}$ Ren Tan, ${ }^{2}$ Mark Hurwitz, ${ }^{1,2}$ Helen Rodgers ${ }^{2}$ and Imogen Mitchell ${ }^{1,2}$ \\ ${ }^{1}$ Medical School, The Australian National University, and ${ }^{2}$ The Canberra Hospital, ACT Health, Canberra, Australian Capital Territory, Australia
}

\author{
Key words \\ palliative care, end-of-life, terminal care, \\ palliative medicine, death.

\section{Correspondence} \\ Brett Scholz, ANU Medical School, \\ The Australian National University, \\ 54 Mills Road, Acton, ACT 2601, Australia. \\ Email: brett.scholz@anu.edu.au
}

Received 11 October 2018; accepted 19 March 2019.

\begin{abstract}
Background: There is a gap in knowledge about the kind and quality of care experienced by hospital patients at the end of their lives.

Aims: To document and compare the patterns in end-of-life care for patients dying across a range of different medical units in an acute care hospital.

Methods: A retrospective observational study of consecutive adult inpatient deaths between 1 July 2010 and 30 June 2014 in four different medical units of an Australian tertiary referral hospital was performed. Units were selected on the basis of highest inpatient death rates and included medical oncology, respiratory medicine, cardiology and gastroenterology/hepatology.

Results: Overall, $41 \%$ of patients died with active medical treatment plans, but significantly more respiratory and cardiology patients died with ongoing treatment (46 and $75 \%$ respectively) than medical oncology and gastroenterology patients (each $27 \%, P<$ $0.05)$. More medical oncology and gastroenterology patients were recognised as dying (92 and $88 \%$ ) compared with $72 \%$ of respiratory and only $38 \%$ of cardiology patients $(P<0.001)$. Significantly, more medical oncology patients were referred to palliative care and received comfort care plans than all other patient groups. However, the rate of non-palliative interventions given in the final $48 \mathrm{~h}$ was not significantly different between all four groups.

Conclusions: There were differences in managing the dying process between all disciplines. A possible solution to these discrepancies would be to create an integrated palliative care approach across the hospital. Improving and reducing interdisciplinary practice variations will allow more patients to have a high-quality and safe death in acute hospitals.
\end{abstract}

\section{Introduction}

Medical advances have facilitated longer life-expectancy but with concomitantly increasing high rates of chronic disease, contributing to the majority of Australian deaths occurring in hospitals. ${ }^{1,2}$ However, surveys reveal most people would prefer to die at home, ${ }^{3}$ and moreover, most patients retain their preference to die at home even as their prognoses worsen. ${ }^{4}$ Therefore, there is uncertainty whether circumstances surrounding in-hospital deaths align with patients' end-of-life wishes, and if quality end-of-life care is consistently and reliably provided in Australian hospitals. ${ }^{1}$

The principal aim of healthcare in Australian hospitals is to cure disease through diagnosis and treatment, and

Funding: None.

Conflict of interest: None. failing this, to sustain life and manage symptoms. ${ }^{5}$ Timely recognition of the dying patient is essential for delivering appropriate end-of-life care. If treatment with curative intent is no longer feasible or realistic, the healthcare team role should ideally change to focus on symptom control to maintain quality of life for as long as possible. These changes need to be made together with patients and families. However, our death-denying culture can militate against acceptance of dying and timely transitions to comfort care. ${ }^{6}$ Evidence suggests some doctors are reluctant to relinquish active intervention even when it is recognised as futile., ${ }^{7,8}$ Overly optimistic patient or family expectations for reversing deterioration may also contribute to providing futile treatment. 9

Failure to recognise death approaching means dying patients may undergo invasive investigations and treatments with no benefit up until death, ${ }^{5,10}$ missing opportunities for referral to hospice or discharge to 
home. ${ }^{11}$ Even when treating teams recognise a patient is approaching death, the prognosis may not always be communicated to patients and their loved ones. ${ }^{12}$ When conversations regarding goals of care, treatment limitations or commencement of palliative care are undertaken, they may occur very late in the dying process. ${ }^{13}$ A lack of timely end-of-life conversations may deny patients the opportunity to prepare for their death or achieve a good death per their own terms. The National Consensus Statement of end-of-life care emphasises the time patients require to address nonhealth related issues, such as religious observances, settling financial affairs and saying goodbyes - essential elements for safe and high-quality end-of-life care. $^{14}$

Historically, end-of-life care research has focused on cancer patient populations, despite other diseases causing more deaths. ${ }^{15}$ More recently, researchers have begun to question what quality of care patients without cancer experience at the end of their lives. International evidence suggests cancer patients receive more frequent and earlier referrals to palliative care, and fewer invasive interventions before death, ${ }^{16,17}$ than people dying from other causes.

This study is a replication and extension of an audit of 100 deaths among patients with oncological or haematological malignancies over 10 years ago at The Canberra Hospital, and followed a more recent hospitalwide pilot study to develop the data collection tool. The overarching aim was to document and explore differences in recent patterns of end-of-life care across a range of medical units within a large Australian tertiary acute care hospital and to explore ways in which different units are meeting best practice standards for end of life care.

\section{Methods}

The ACT Health Human Research Ethics Committee approved the study and waived the need for consent. An audit of 529 consecutive adult inpatient deaths occurring between 1 July 2010 and 30 June 2014, in the four medical units with the highest number of deaths (medical oncology $(n=226)$, respiratory medicine $(n=109)$, cardiology $(n=112)$ and gastroenterology/hepatology $(n=$ 82)) was conducted. Four years of data were determined to reflect the end of life care provided in each discipline. The audit was performed using a data extraction tool developed for the more recent hospital-wide pilot study on end-of-life research in acute settings. These units were selected as they had the hospital's highest total number of in-patient ward deaths during the 4-year study period. The hospital is a tertiary, universityaffiliated hospital with 600 beds and services a population of 660000 . The medical oncology unit had 10 specialist staff members. Respiratory medicine had 10 staff specialists, cardiology had 9 staff specialists, and the gastroenterology/hepatology unit had 15 staff specialists.

Inclusion criteria for the audit were deaths on wards occurring between 1 July 2010 and 30 June 2014, patient age 18+ years; deaths occurring in the Emergency Department and Intensive Care Unit (ICU) were excluded. All consecutive deaths under consultants from each of the four units were identified by the hospital clinical records department. Data were extracted from the clinical records information system for each patient's final hospital admission, noting that pre-transfer notes for patients referred to the hospital were not available to the researchers.

The primary outcome measure was the number of patients receiving ongoing acute treatment or interventions until time of death. The audit tool collected these data through application to clinical notes and recording active medical management during the final 2 days of life was defined as including i.v. antibiotics, blood products, vasoactive medications, dialysis, blood tests, medical imaging, chemotherapy, radiotherapy, intubation/continuous positive airway pressure/bilevel positive airway pressure ventilation, invasive interventions, such as operations/cardiac catheterisation/ endoscopy, ICU/high dependency unit admission and medical emergency team calls.

Clinical notes were reviewed for evidence of pre-existing advance care plans, resuscitation plans, medical interventions within $48 \mathrm{~h}$ of death, written recognition of dying, palliative care referral and comfort care measures. Four coders independently extracted data for each unit, and 40 records were randomly assessed for inter-rater reliability by an independent coder - the researcher who had developed the data extraction tool for a pilot study. The joint probability of agreement was high, ranging between 94 and $99 \%$ across the four datasets, with no systematic variations observed.

Data extraction and analysis occurred between December 2015 and July 2016. Data were entered into a custom-built Microsoft Access database (2013, Seattle, WA, USA) and analysed in Excel (2016 Microsoft, Washington, DC, USA). The chi-squared test was used to identify significant differences in proportions between the Medical Oncology patient group and each of the three other patient groups. 


\section{Results}

\section{Primary outcome measure: active treatment versus comfort care at death}

All data are presented in Table 1. For all patients, we looked for documented evidence of whether the goals of care shifted from active medical management aimed at reversing current deterioration to comfort care intended to palliate symptoms.

Most medical oncology and gastroenterology patients were transitioned to a comfort care plan, and were significantly less likely to have active treatment/ interventions at time of death. In both of these cohorts, $27 \%$ died with active treatments in place $(60 / 226$ and $22 / 82$ respectively). Rates of active medical intervention at time of death were higher in the other two units: nearly half of respiratory patients $(46 \%, 50 / 109, P<$ $0.001)$ and three-quarters of cardiology patients $(75 \%$, $84 / 112, P<0.001)$. Nearly four in 10 cardiology deaths in this study occurred within $48 \mathrm{~h}$ of admission, which is a significantly higher proportion than the other three units $(39 \%, 68 / 112, P<0.001)$.

\section{Secondary outcome measures}

\section{Advance directives and resuscitation planning}

Overall, few patients had advance care directives (ACD) or enduring power of attorney (EPOA) documented on admission. Only $9 \%$ of medical oncology patients had a documented EPOA (21/226), significantly less than cardiology $(17 \%, 19 / 112, P=0.040)$, gastroenterology $(20 \%, 16 / 82, P<0.001)$ and respiratory patients $(24 \%$, $26 / 109, P<0.001)$. Few patients in any group had a documented ACD, at 4\% among cardiology (5/112) and gastroenterology $(3 / 82)$ patients, $5 \%$ among medical oncology (12/226) patients, although significantly more respiratory patients $(14 \%, 15 / 109, P=0.008)$.

A cardiopulmonary resuscitation (CPR) plan was documented during final admission for most patients $(87 \%, 459 / 529)$. The rate of resuscitation planning was the highest among medical oncology patients $(98 \%$; $221 / 226)$. While the majority of gastroenterology patients' records contained a resuscitation plan, this was significantly lower than in the medical oncology unit (91\%, 75/82, $P<0.001)$. Only approximately threequarters of respiratory $(76 \%, 83 / 109, P<0.001)$ and cardiology $(71 \%, 80 / 112, P<0.001)$ patients had resuscitation plans in place, despite having the oldest age profiles of the four cohorts (see Table 1). In addition to CPR status, many plans documented forms of ventilation and ICU transfer status, but there was wide variability in what treatment options were specified as appropriate or to be withheld.

\section{Recognition of dying, interventions, comfort care planning and referral to palliative care}

Most medical oncology $(92 \% ; 208 / 226)$ and gastroenterology $(88 \% ; 72 / 82, P=0.254)$ patients' clinical notes contained documentation suggesting recognition the patient was dying. Significantly fewer respiratory patients' notes contained written statements recognising

Table 1 Patient characteristics and outcome measures

\begin{tabular}{|c|c|c|c|c|c|c|c|c|}
\hline & \multirow{2}{*}{$\frac{\text { Medical oncology }}{n(\%)}$} & \multicolumn{2}{|c|}{ Respiratory medicine } & \multicolumn{2}{|c|}{ Cardiology } & \multicolumn{2}{|c|}{$\begin{array}{c}\text { Gastroenterology and } \\
\text { hepatology }\end{array}$} & \multirow[t]{2}{*}{ Total } \\
\hline & & $n(\%)$ & Significant $P$ & $n(\%)$ & Significant $P$ & $n(\%)$ & Significant $P$ & \\
\hline \multicolumn{9}{|l|}{ Patient characteristics } \\
\hline Patients & 226 & 109 & & 112 & & 82 & & 529 \\
\hline Admissions $\geq 48 \mathrm{~h}$ & $196(87)$ & $90(83)$ & 0.313 & $68(61)$ & $<0.001$ & $70(85)$ & 0.759 & 424 \\
\hline Median age (years) & 66 & 78 & & 80 & & 71 & & \\
\hline Males & $116(51)$ & $70(64)$ & 0.026 & $77(69)$ & $<0.001$ & $62(76)$ & $<0.001$ & 325 \\
\hline \multicolumn{9}{|l|}{ Primary outcome } \\
\hline Active medical treatment at death & $60(27)$ & $50(46)$ & $<0.001$ & $84(75)$ & $<0.001$ & $22(27)$ & 0.961 & 216 \\
\hline \multicolumn{9}{|l|}{ Secondary outcome measures } \\
\hline Enduring power of attorney & $21(9)$ & $26(24)$ & $<0.001$ & $19(17)$ & 0.040 & $16(20)$ & $<0.001$ & 82 \\
\hline Advance care plan & $12(5)$ & $15(14)$ & 0.008 & $5 \dagger(4)$ & & $3 \dagger(4)$ & & 35 \\
\hline Resuscitation plan & $221(98)$ & $83(76)$ & $<0.001$ & $80(71)$ & $<0.001$ & $75(91)$ & $<0.001$ & 459 \\
\hline Recognition of dying & $208(92)$ & $78(72)$ & $<0.001$ & $42(38)$ & $<0.001$ & $72(88)$ & 0.254 & 377 \\
\hline Comfort care plan & $194(86)$ & $74(68)$ & $<0.001$ & $38(34)$ & $<0.001$ & $62(76)$ & 0.034 & 368 \\
\hline Palliative care referral & $194(86)$ & $63(58)$ & $<0.001$ & $21(19)$ & $<0.001$ & $54(66)$ & $<0.001$ & 332 \\
\hline Interventions in last $48 \mathrm{~h}:$ & $147(75)$ & $62(69)$ & 0.279 & $49(68)$ & 0.633 & $42(60)$ & 0.018 & 300 \\
\hline
\end{tabular}

$\dagger$ Cell sizes too small for chi-square test assumptions. $\$$ Denominator for percentage calculation contains only patients admitted for $\geq 48 \mathrm{~h}$. Bold represents significant $P$-values. 
death was likely $(72 \%, 78 / 109, P<0.001)$, though the majority were noted as being in terminal decline.

Cardiology patients were the least likely to have recognition of dying charted in their notes $(38 \%, 42 / 112, P<$ $0.001)$. However, this did not mean the treating team was unaware the patient might soon die - only that it was not written explicitly in the medical record. Despite the low proportion of cardiology patients receiving referrals to the palliative care service $(19 \%, 21 / 112, P<$ $0.001)$, more cardiology patients had comfort care plans prepared than had received palliative care referrals $(34 \%, 38 / 112, P<0.001)$. Rates of referral to palliative services and charting of comfort care plans were still the lowest for this cohort of patients.

Patients under the care of medical oncology were significantly more likely to have comfort care plans charted $(86 \%, 194 / 226)$, as well as receive palliative care service referrals $(86 \%)$, than those in the other three units. Among gastroenterology patients, 76\% (62/82, $P=$ $0.034)$ had comfort care plans documented and twothirds $(66 \% ; 54 / 82, P<0.001)$ received palliative care referrals, while among respiratory patients, $68 \%$ (74/109, $P<0.001)$ had comfort care plans and 58\% (63/109, $P<0.001)$ were referred to palliative care. When comfort care goals were documented, then several forms of active medical management were less likely to be used during the patients' final 2 days of life, including vasoactive medication, dialysis, intubation and medical emergency team calls.

When patients dying within $48 \mathrm{~h}$ of hospital admission were excluded, we found $63 \%(42 / 70)$ of gastroenterology patients received active medical interventions, which was a significantly lower rate than the $75 \%$ (147/196, $P=0.018$ ) observed among medical oncology patients. There were no significant differences in the rate of late medical interventions (in the last $48 \mathrm{~h}$ before death) used between the medical oncology and cardiology $(68 \%, 49 / 72, P=0.633)$ or respiratory $(69 \%, 62 / 90, P=$ $0.279)$ units. Overall, however, the majority of patients in this study still received some non-comfort care management in their last $48 \mathrm{~h}$ of life.

\section{Discussion}

This study reports end-of-life care for 529 patients who died in medical oncology, respiratory, cardiology and gastroenterology medical units at The Canberra Hospital over a 4-year period ending 30 June 2014. There was considerable variation in rates of active medical management at time of death, with medical oncology and gastroenterology patients being least likely to receive potentially inappropriate active medical interventions at time of death. Significantly more medical oncology patients had comfort care plans documented compared to the other units. The majority of medical cardiology patients received active medical management at death, though one-third had comfort care plans charted at the same time. Some of the differences in patients' experience of dying between these medical units might reflect differences in clinicians' understandings of what specialist palliative care can offer and when to initiate palliative care. Given the historical links between palliative care and oncology units (and the common misconception that palliative care is for oncology patients ${ }^{18}$ ), engagement in palliative care by other disciplines may be limited, particularly if there are inadequate resources.

These findings extend current literature suggesting treatment and investigations at the end-of-life may result from a belief the patient could yet recover, or through a lack of physician confidence in ceasing active management and implementing comfort care. ${ }^{7,19}$ The findings of the current study suggest recognition of terminal decline occurred close to time of death. This is evidenced by the majority of patients in all units receiving some active medical management within $48 \mathrm{~h}$ of death, with no significant difference in intervention rates across the units. Furthermore, not all patients with documented plans for comfort care had their care type changed prior to death. However, patient and family expectations of prognosis and treatment were not investigated in this study, and may have a bearing on the interventions used at the end of life due to overestimated chances of recovery. ${ }^{9}$

The findings of this study have particular implications for practice and education. First, in terms of practice, these findings suggest it is important for clinicians to take into account the age of patients in deciding whether to commence comfort care. Previous research in Scotland suggests that older people receive less palliative care than younger people, ${ }^{20}$ consistent with this study demonstrating that patients in cardiology receive the least comfort care despite having the oldest age profile.

Second, in terms of education, the findings of the current study suggest that clinicians from across medical disciplines might benefit from more training in understanding how to recognise when patients would benefit from specialist and non-specialist palliative care. The variation in care given across disciplines might mean that there are currently inconsistent levels of understanding of palliative care across disciplines. The quality of care provided - and in turn patients' and carers' hospital experiences - would benefit from better understandings of when palliative care is appropriate.

This study contributes to the growing evidence of variability in quality of end-of-life care across medical disciplines. ${ }^{16}$ One of the strengths of the study is that it 
consecutively sampled all in-patient deaths in the four medical units with the highest numbers of deaths in a 4-year period, using a standardised data collection tool informed by prior research. ${ }^{21}$

There are also some weaknesses of the current study. First, as a retrospective observational study, this work assumes thorough and accurate clinical records; the potential for incomplete and inaccurate notes to bias findings cannot be quantified. Second, the list of inventions determined to be medical management was defined in advance of data collection, and thus assumes inventions, such as i.v. antibiotics and fluids were noncomfort measures. It is possible treating clinicians may have provided some of these measures as relieving symptoms, or to help families feel better about the care of loved ones in their final hours. Last, data collection was undertaken in one tertiary Australian hospital, potentially limiting generalisability of results. However, the findings correlate with patterns in local and international research that the transition to palliative care occurs more frequently for patients with malignancies. ${ }^{16,17,22,23}$ Thus, it is likely the conclusions drawn from the study relate to end-of-life care more broadly.

Given our findings relate to the difficulty to recognise terminal decline, future studies should assess whether improvements in end-of-life care can be made through the adoption of clinical tools to facilitate terminal decline recognition, ${ }^{24}$ such as the Supportive and Palliative Care Indicators Tool. ${ }^{25}$

\section{Conclusion}

This study found recognition of terminal decline and the end-of-life care provided to patients dying in an acute hospital varies across medical units. The variations in the dying process between medical disciplines provide an understanding of what is needed to ensure compliance with the recommendations of the National Consensus Statement: Essential Elements of Safe and High-Quality Endof-Life Care. ${ }^{14}$ The findings of the current study suggest that best practice for end-of-life care need to be implemented more uniformly across health disciplines, and emphasise the need for leadership to reduce variability in the quality and timeliness of end-of-life care across hospital units. The development of guidelines encouraging early engagement with the provision of palliative care, specialist or otherwise, for all patients with a potentially life-limiting chronic disease would allow for timely end of life care in their preferred place of care.

\section{References}

1 Australian Commission on Safety and Quality in Health Care. Safety and Quality of End-of-life Care in Acute Hospitals: A Background Paper. Sydney: The Commission; 2013.

2 Australian Institute of Health and Welfare. Australia's Health 2016. Canberra: The Institute; 2016.

3 Gomes B, Calanzani N, Gysels M, Hall S, Higginson IJ. Heterogeneity and changes in preferences for dying at home: a systematic review. BMC Palliat Care 2013; 12: 7 .

4 Foreman LM, Hunt RW, Luke CG, Roder DM. Factors predictive of preferred place of death in the general population of South Australia. Palliat Med 2006; 20: 447-53.

5 Middlewood S, Gardner G, Gardner A. Dying in hospital: medical failure or natural outcome? J Pain Symptom Manage 2001; 22: 1035-41.

6 The Royal Australasian College of Physicians (RACP). Improving Care at the End of Life: Our Roles and Responsibilities. Sydney: RACP; 2016.

7 Gott M, Ingleton C, Bennett MI, Gardiner C. Transitions to palliative care in acute hospitals in England: qualitative study. Br Med J 201 1; 342: d 1773 .

8 Willmott L, White B, Close E, Gallois C, Malcolm P, Graves N et al. Futility and the law: knowledge, practice and attitudes of doctors in end of life care. QUT Law Rev 2016; 16: 55-75.

9 Hole B, Salem J. How long do patients with chronic disease expect to live? A systematic review of the literature. $B M J$ Open 2016; 6: e012248.

10 Veerbeek L, Van Zuylen L, Swart SJ, Jongeneel G, Van der Maas PJ, Van der Heide A. Does recognition of the dying phase have an effect on the use of medical interventions. $J$ Palliat Care 2008; 24: 94-9.

11 Houttekier D, Cohen J, Pepersack T, Deliens L. Dying in hospital: a study of incidence and factors related to hospital death using death certificate data. Eur $J$ Public Health 2014; 24: 751-6.

12 Al-Qurainy R, Collis E, Feuer D. Dying in an acute hospital setting: the challenges and solutions. Int J Clin Pract 2009; 63: 508-15.

13 Boyd K, Murray SA. Recognising and managing key transitions in end of life care. Br Med J 2010; 341: c4863.

14 Australian Commission on Safety and Quality in Health Care. National
Consensus Statement: Essential Elements for Safe and High-Quality End-of-Life Care. Sydney: The Commission; 2015.

15 Australian Bureau of Statisitics. Cause of Death, Australia, 2015. Canberra: The Bureau; 2016.

16 Wachterman MW, Pilver C, Smith D, Ersek M, Lipsitz SR, Keating NL. Quality of end-of-life care provided to patients with different serious illnesses. JAMA Intern Med 2016; 176: 1095-102.

17 Barnato AE, Cohen ED, Mistovich KA, Chang C-CH. Hospital end-of-life treatment intensity among cancer and non-cancer cohorts. J Pain Symptom Manage 2015; 49: 521-9.e5.

18 Ward $C$. The need for palliative care in the management of heart failure. Heart 2002; 86: 294-8.

19 Cairns W. We can improve how we deal with death, now. MJA InSight 2016 [cited 2018 Aug 7]. Available from URL: https://insightplus.mja.com.au/ 2016/10/we-can-improve-how-we-dealdeath-now/.

20 Lloyd A, Kendall M, Carduff E, Cavers D, Kimbell B, Murray S. Why do older people get less palliative care than younger people? Eur J Pallat Care 2016; 23: 132-7.

21 Batten M, Nguyen B, Burke B, Harryanto H, Mitchell I, Davis A. End- 
of-life care of oncology inpatients: are we getting it right? Asia Pac J Clin Oncol 2018; 14: e528-34.

22 Mitchell GK, Johnson CE, Thomas K, Murray SA. Palliative care beyond that for cancer in Australia. Med J Aust 2010; 193: 124-6.

23 Fins JJ, Miller FG, Acres CA,

Bacchetta MD, Huzzard LL, Rapkin BD.
End-of-life decision-making in the hospital: current practice and future prospects. J Pain Symptom Manage 1999; 17: 6-15.

24 Amblàs-Novellas J, Murray SA, Espaulella J, Martori JC, Oller R, Martinez-Muñoz $\mathrm{M}$ et al. Identifying patients with advanced chronic conditions for a progressive palliative care approach: a cross-sectional study of prognostic indicators related to end-oflife trajectories. BMJ Open 2016; 6: e012340.

25 NHS Lothian, University of Edinburgh. Supportive and Palliative Care Indicators Tool (SPICT). Edinburgh: NHS Lothian; 2016.

\section{Improving survival in patients with hepatocellular carcinoma related to chronic hepatitis $C$ and $B$ but not in those related to non-alcoholic steatohepatitis or alcoholic liver disease: a 20-year experience from a national programme}

Ibrahim Hassan (1) and Edward Gane

New Zealand Liver Transplant Unit (NZLTU), Auckland City Hospital, Auckland, New Zealand

Key words

hepatocellular carcinoma (HCC), HCC surveillance, HBV-related HCC, HCV-related, NASH-related HCC.

\section{Correspondence}

Ibrahim Hassan, Department of

Gastroenterology and Hepatology, Auckland City

Hospital, 2 Park Road, Grafton, Auckland 1023,

New Zealand.

Email: ibrahim20.hassan20@gmail.com

Received 25 September 2018; accepted 15 March 2019

\begin{abstract}
Background: Hepatocellular carcinoma (HCC) is the most rapidly increasing cause of cancer mortality in New Zealand due to endemic hepatitis B virus (HBV) infection and recent hepatitis $\mathrm{C}$ virus (HCV) and obesity epidemics.

Aim: To describe the changing landscape of HCC referred to a national HCC service over a 20-year period, including trends in underlying liver disease, screening uptake and access to curative treatments, and to determine the impact of screening on outcomes with a comparison between screened detected and non-screened detected cases. Methods: All newly diagnosed cases of HCC referred to New Zealand Liver Transplant Unit between 1998 and 2017 were included. Data on patient demographics, liver disease aetiology, screening status and treatment modalities were collected.

Results: HCC diagnosis rates have increased from 24 cases in 1998 to 250 in 2017, an increase of $20 \%$ per annum. The total of 1985 HCC cases was divided into three cohorts (Era 1: 1998-2009; Era 2: 2009-2014; Era 3: 2014-2017), each comprising 661-662 patients. During the study period, overall survival improved $(P=0.005)$. The proportion with screen-detected HCC was similar across the three cohorts ( $44 \%$ in Era 1, $42 \%$ in Era 2 and $47 \%$ in Era 3). Five- and 10-year survival was higher in screen-detected cases (49 and $43 \%$ ) than in non-screen detected cases (14 and 10\%), $P<0.0001$. Survival was higher in patients with HCV and HBV than in those with non-alcoholic steatohepatitis (NASH) or alcoholic liver disease (ALD) - 5 and 10-year survival was 40 and $34 \%$ in HCV-HCC, 30 and $26 \%$ in HBV-HCC, 15 and $14 \%$ in NASH-HCC, 13 and $10 \%$ in ALD-HCC, $P<0.0001$.

Conclusion: Better outcomes in patients with HBV- or HCV-related HCC than in those with NASH-related or ALD-related HCV may reflect better screening uptake and better access to curative therapies.
\end{abstract}

Funding: None.

Conflict of interest: None.

\section{Introduction}

Liver cancer is the second leading cause of cancer-related mortality worldwide accounting for 788000 deaths in 